fibrinogen, HbA1c increased significantly as the numbers of MetSyn components increased whereas levels of ApoA1 decreased $(p<0.05)$. Conclusions Our results suggest that increasing numbers of metabolic syndrome components are associated with an elevated level of both markers of chronic low-grade inflammation and intermediate disease. Our findings support previous work showing that persons with MetSyn are a clinically relevant population with underlying pathogenesis that could benefit from early treatment.

\section{P2-34 ASSOCIATION OF ANTHROPOMETRIC AND LIFESTYLE FACTORS WITH PROSTATE SPECIFIC ANTIGEN (PSA) TRAJECTORIES IN MEN WITH LOCALISED PROSTATE CANCER UNDERGOING ACTIVE MONITORING}

doi:10.1136/jech.2011.142976h.70

${ }^{1} \mathrm{~A}$ Burton, ${ }^{* 1} \mathrm{R}$ Martin, ${ }^{1} \mathrm{~J}$ Holly, ${ }^{2} \mathrm{~F}$ Hamdy, ${ }^{3} \mathrm{D}$ Neal, ${ }^{1} \mathrm{~J}$ Donovan, ${ }^{1} \mathrm{~K}$ Tilling. ${ }^{1}$ University of Bristol, Bristol, UK; ${ }^{2}$ University of Oxford, Oxford, UK; ${ }^{3}$ University of Cambridge, Cambridge, UK

Introduction Widespread use of Prostate-specific Antigen (PSA) testing has lead to a rapid increase in the identification of low risk prostate cancer, with PSA trajectories often used to monitor tumour progression after diagnosis. Here we develop novel age-specific multi-level growth curves to assess the effects of lifestyle and anthropometric measures on PSA trajectories.

Methods Serial PSA measures from 513 men aged $50-70$ years with localised prostate cancer undergoing active monitoring were used to develop models. Gleason score, height, weight, body mass index, waist, waist to hip ratio, smoking and alcohol consumption were each added to the basic age-specific growth curve model as explanatory variables.

Results The basic growth curve gave an average PSA at age 50 of $2.10 \mathrm{ng} / \mathrm{ml}$ (95\% CI 1.85 to 2.38 ) and a yearly increase in PSA of $1.07 \mathrm{ng} / \mathrm{ml}$ (95\% CI 1.06 to 1.08 ) (or 7\%; 95\% CI 6\% to 8\%). In these preliminary findings, Gleason score at baseline was strongly associated with PSA growth: the yearly increase in PSA was 3.4\% greater ( $95 \%$ CI $1 \%$ to $6 \%$ ) for men with Gleason of 7 or greater vs those with Gleason 6 or less. Current smoking was positively associated with PSA change: the yearly increase in PSA was $3.2 \%$ greater $(95 \%$ CI $0 \%$ to $7 \%$ ) for current vs never smokers. No other factors were strongly associated with either initial PSA or yearly increase in PSA. Conclusions Smoking status may be associated with PSA trajectory in men being followed up by active monitoring but other lifestyle and anthropometric factors have little association.

\section{P2-35 OUTCOMES OF SMALL FOR GESTATIONAL AGE (SGA) BIRTH AS A PLACENTA ASSOCIATED SYNDROME}

doi:10.1136/jech.2011.142976h.71

\begin{abstract}
1,2M K Campbell, * 1,2W R Avison, 1,2K N Speechley. 'Schulich School of Medicine and Dentistry, The University of Western Ontario, London, Ontario, Canada; ${ }^{2}$ The Children's Health Research Institute, London, Ontario, Canada
\end{abstract}

Introduction Infants born small for gestational age (SGA) are at higher risk for morbidity. This analysis explored whether increased risk is attributable to SGA or its causes.

Methods A population-based cohort $(n=2357)$ was recruited at 10-22 weeks gestation. We collected prenatal data by questionnaire, perinatal data from hospital records, and follow-up data by telephone survey 2-4 years later. Outcomes included neonatal admission to special care nursery (NASC), neonatal Apgar score $<7$ at 1 min (Apgar1) and, hospital admission (HA) during the 12 months preceding the follow-up interview. Analyses included multivariable logistic regression.
Results Preeclampsia had statistically significant multivariable associations with NASC and Apgar1; OR's (95\% CI's) were 2.0 (1.0 to 5.0 ) and 2.4 (95\% CI 1.3 to 4.3), respectively. Apgar1 was also associated with young maternal age and obesity. In secondary analysis restricted to term deliveries, threatened preterm labour (TPL) had OR's of $3.9(1.6,9.3)$ and $2.2(1.2,4.2)$, respectively, for NASC and Apgar1. TPL displaced preeclampsia from the model indicating correlation. Since recent literature suggests that preeclampsia, TPL, and SGA have common aetiologies, we combined these into a single construct, Placenta Associated Syndromes (PAS) (Alilu et al 2010). The OR's of PAS with NSC and Apgar1 were 3.9 $(1.6,9.3)$ and $2.2(1.2,4.2)$, respectively. HA had multivariable associations with preeclampsia and maternal depression (CES$\mathrm{D}>24)$ with OR's of $2.6(1.1,6.1)$ and $2.9(1.3,6.4)$. SGA was not independently associated with outcomes studied.

Conclusion We conclude that SGA is a marker for placental dysfunction, which is the true risk factor for the outcomes studied.

\section{P2-36 BODY MASS INDEX AND RISK OF INCIDENT ISCHAEMIC HEART DISEASE IN WOMEN: A PROSPECTIVE COHORT STUDY}

doi:10.1136/jech.2011.142976h.72

D Canoy, ${ }^{*}$ B J Cairns, A Balkwill, G K Reeves, J Green, V Beral. Cancer Epidemiology Unit, University of Oxford, Oxford, UK

Background Various studies have examined body mass index in relation to mortality due to ischaemic heart disease (IHD), but few data are available on incident IHD including non-fatal events particularly in women.

Methods We used multivariate Cox regression, taking into account confounding and mediating factors, to examine the association between body mass index and first IHD event (ascertained from hospital records and death registration) in the Million Women Study, a population-based prospective cohort of middle age British women.

Results In this cohort of women aged $50-70$ years, there were 48895 incident IHD events during 10.7 million person-years of follow-up, including 5108 deaths with IHD identified as the primary cause. IHD incidence rate was 2.1 per 100 women per 5 years. Mean measured BMI (SD) was $26.7(4.7) \mathrm{kg} / \mathrm{m}^{2}$. Risk for IHD increased with higher BMI $(p<0.001)$. The large number of incident IHD events in this cohort makes it possible to examine in detail possible effect modification by other risk factors, including comparing associations between current and never smokers, or by socioeconomic status or physical activity level.

Conclusion Non-fatal IHD adds considerably to the burden of heart disease. In this very large prospective study, incident IHD in women was strongly associated with excess weight.

\section{P2-37 ASSOCIATION BETWEEN SLEEP DURATION AND ALL- CAUSE MORTALITY IN OLD AGE: 9-YEAR FOLLOW-UP OF THE BAMBUÍ COHORT STUDY, BRAZIL}

doi:10.1136/jech.2011.142976h.73

\begin{abstract}
${ }^{1,2}{ }^{2}$ Castro-Costa, ${ }^{* 2} \mathrm{M}$ Dewey, ${ }^{2} \mathrm{C}$ Ferri, ${ }^{1,3} \mathrm{E}$ Uchoa, ${ }^{1} \mathrm{~J}$ Firmo, ${ }^{4} \mathrm{~F}$ Rocha, ${ }^{2} \mathrm{M}$ Prince ${ }^{1,3} \mathrm{M}$ F Lima-Costa, ${ }^{2} \mathrm{R}$ Stewart. ${ }^{1}$ Centro de Pesquisa Rene Rachou, Fiocruz, Belo Horizonte, Minas Gerais, Brazil; ${ }^{2}$ King's College London, Institute of Psychiatry, London, UK; ${ }^{3}$ Federal University of Minas Gerais Medical School, Belo Horizonte, Minas Gerais, Brazil; ${ }^{4}$ IPSEMG, Belo Horizonte, Minas Gerais, Brazil
\end{abstract}

Introduction This study investigates the association of sleep duration with risk of all-cause mortality among elderly Brazilians using data from a 9-year population-based cohort study. 